\title{
Daily home spirometry to detect early steroid treatment effects in newly treated pulmonary sarcoidosis
}

\author{
To the Editor:
}

Prednisone is the mainstay of sarcoidosis treatment. However, prednisone treatment optimisation is warranted, since prolonged high-dose prednisone therapy is associated with burdensome and harmful side-effects $[1,2]$. Early prednisone dose tapering has the potential to reduce side-effects. Gaining insight in the early treatment response can help to determine when tapering could be initiated. To date, there are no prospective studies that look at early treatment response to prednisone in sarcoidosis by monitoring clinical symptoms and daily patient-administered lung function. Therefore, we initiated a multicentre, prospective and observational study with daily home spirometry to detect early steroid treatment effects in newly treated pulmonary sarcoidosis (Dutch National Trial Register NTR4328; www.trialregister.nl/trialreg).

Treatment-naïve sarcoidosis patients in whom prednisone therapy was about to be initiated for a pulmonary indication were eligible. Patients were intended to be treated with the following prednisone regimen: 4 weeks $40 \mathrm{mg} \cdot \mathrm{day}^{-1}$, 2 weeks $30 \mathrm{mg} \cdot \mathrm{day}^{-1}$, 2 weeks $20 \mathrm{mg} \cdot \mathrm{day}^{-1}$, 2 weeks $15 \mathrm{mg} \cdot \mathrm{day}^{-1}$, 2 weeks $10 \mathrm{mg} \cdot \mathrm{day}^{-1}$ (unless the treating physician decided that the clinical situation demanded deviation). During these 3 months, daily home monitoring of forced vital capacity (FVC) was performed by the patient on a calibrated hand-held spirometer (Micro Diary; Carefusion, Hoechberg, Germany). Additionally, patients were asked to fill out a Medical Research Council (MRC) dyspnoea and Fatigue Assessment Scale (FAS) score at the end of each week in a diary. Data are presented as mean \pm sD unless stated otherwise. More details on the study design can be found in the trial register online.

The study group consisted of 21 patients (13 male and eight female; age $43 \pm 11$ years). The majority of the patients $(76 \%)$ were diagnosed with Scadding stage II sarcoidosis. Routine in-hospital lung function monitoring showed a significant FVC increase following treatment from $69.7 \pm 13.9$ to $81.5 \pm 13.7 \%$ predicted at month 1 (mean change $11.8 \pm 9.2, \mathrm{p}<0.001$ ) (figure 1a). A smaller FVC improvement was observed between month $1(81.5 \pm 13.7)$ and month $3(84.8 \pm 13.2)$ (mean change $3.3 \pm 6.4, \mathrm{p}=0.039)$ (figure 1a). The estimated mean FVC change obtained using daily home spirometry over time was calculated using a fixed effect model, meaning that the regression lines of all 21 individual patients were incorporated (figure 1c). A maximal mean increase of 9.7 (95\% CI 8.4-10.9) \% pred FVC was estimated after treatment initiation. Interestingly, a plateau of FVC increase was observed at 24 days (95\% CI 14-33) and $90 \%$ of the total FVC increase was already reached by day 18 (95\% CI 10-28).

Together, these data show that most of the improvement in FVC occurs within 2-3 weeks after prednisone therapy initiation in a cohort of newly treated sarcoidosis patients.

Our results are in line with other studies that have suggested that the major increase in FVC during prednisone treatment in sarcoidosis occurs within 1 month [3, 4]. However, these studies were either performed retrospectively in patients experiencing an exacerbation who previously were shown to be responsive to prednisone and/or evaluated changes on in-hospital lung function equipment at pre-determined time-points, possibly missing early and daily changes in FVC $[3,4]$.

No single clinical test has been found to accurately assess disease burden in pulmonary sarcoidosis [5]. Therefore, it is important to evaluate whether other outcomes improve as well. Multilevel analyses of the

@ERSpublications

The major treatment effect of prednisone on FVC is reached within 2 to 3 weeks in newly treated sarcoidosis patients http://ow.ly/3A3E30h5ZuT

Cite this article as: Broos CE, Wapenaar M, Looman CWN, et al. Daily home spirometry to detect early steroid treatment effects in newly treated pulmonary sarcoidosis. Eur Respir J 2018; 51: 1702089 [https:// doi.org/10.1183/13993003.02089-2017]. 

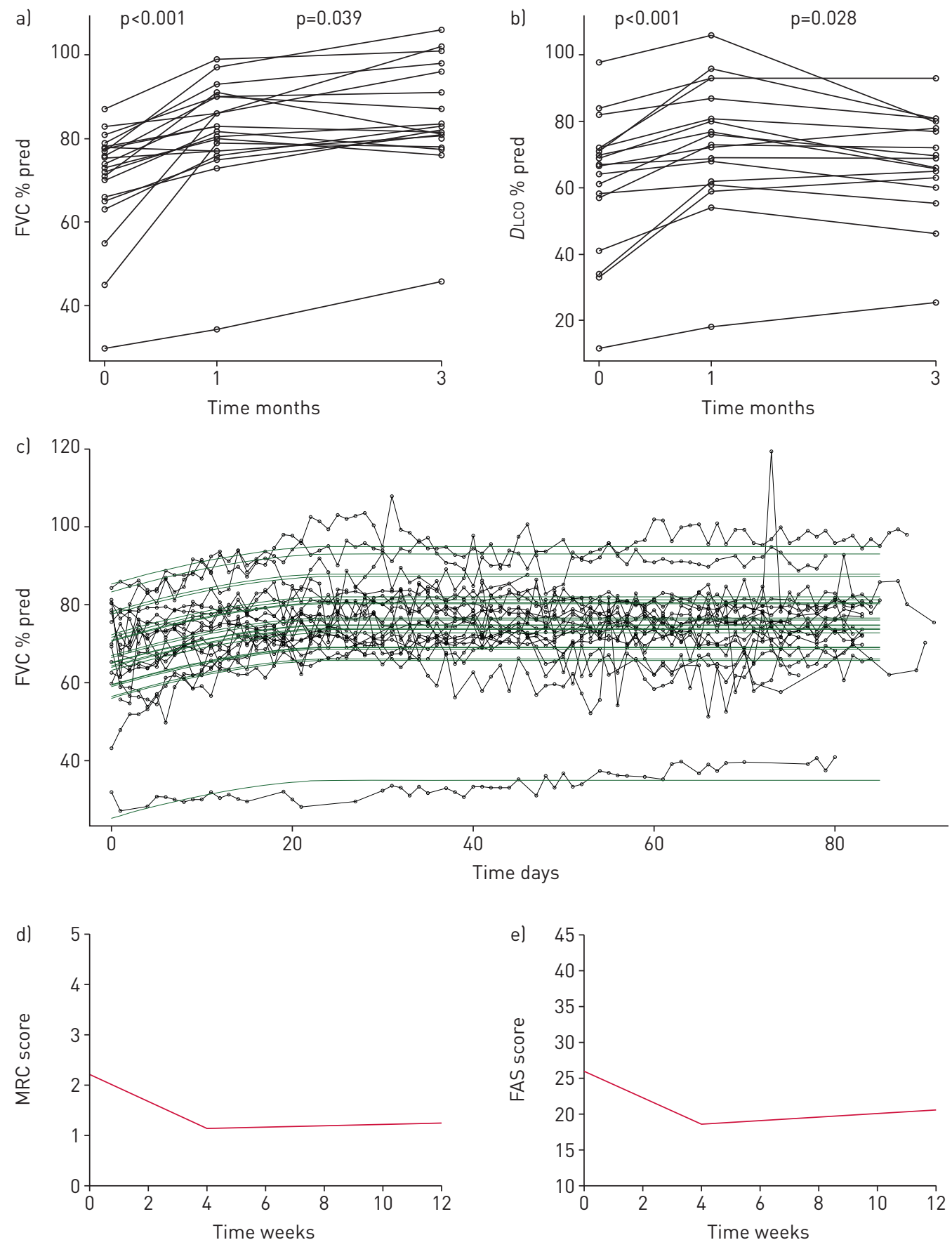

FIGURE 1 a) In-hospital clinical monitoring of forced vital capacity (FVC; n=19) and b) diffusing capacity of the lung for carbon monoxide ( $\mathrm{LLCO}$ ) (corrected for haemoglobin levels; $\mathrm{n=17}$ ) values at baseline, month 1 and month 3 during prednisone treatment of patients with data available at all three time-points. Lines represent paired results from one patient. Significance was determined using a two-tailed paired t-test. c) Daily best FVC measurements recorded on the home spirometer (Micro Diary; Carefusion) per individual patient over time (in days) as \% predicted, including regression lines of all 21 individual patients. Daily FVC measured during home monitoring was used as the outcome in a fixed effect model (fixed effect nonlinear least squares model). Regression line of all d) weekly Medical Research Council (MRC) dyspnoea scores and e) Fatigue Assessment Scale (FAS) scores filled out in a home diary per individual patient over time (in weeks). A multilevel model was used that modelled linear splines with a knot at 4 weeks.

weekly filled out MRC scores showed that dyspnoea symptoms decreased significantly with 0.3 (95\% CI $0.2-0.4)$ MRC points per week $(\mathrm{p}<0.001)$, stabilising after 1 month (figure $1 \mathrm{~d})$. Similarly, FAS scores decreased significantly with 1.8 (95\% CI 1.5-2.2) FAS points per week $(\mathrm{p}<0.001)$, stabilising after 1 month 
(figure 1e). Importantly, this is the first study to evaluate early therapy effects on dyspnoea (MRC) and fatigue (FAS) [6], and the early improvements observed strengthen our home spirometry data.

In a sub-cohort of patients, participating at a select number of study sites, diffusing capacity of the lung for carbon monoxide (DLCO) (corrected for haemoglobin level) was measured. A significant increase in DLCO $\%$ pred was observed within 1 month from $60.4 \pm 20.6$ to $72.5 \pm 19.7$ (mean change: $12.0 \pm 8.5, \mathrm{p}<0.001$ ). However, DLCO decreased somewhat again between month 1 and 3 to $67.5 \pm 15.6$ (mean change $-5.0 \pm 8.5$, $\mathrm{p}=0.028$ ) (figure $1 \mathrm{~b}$ ). These data may indicate subclinical worsening of the disease during prednisone tapering, as DLCO measurements may reflect earlier interstitial changes in sarcoidosis [7]. However, we cannot be certain that this is a relevant change, as reproducibility of DLCO measurements is well known to be lower than FVC measurements, and only a major change ( $>15 \%)$ in DLCO has been suggested to reliably exclude confounding by measurement variation [5]. Furthermore, daily home spirometry and weekly dyspnoea and fatigue scores showed that the initial improvement was retained in the majority of patients up to 3 months while prednisone dose was tapered. In addition, inflammatory markers that are thought to reflect disease activity in sarcoidosis, such as angiotensin-converting enzyme and soluble interleukin-2 receptor $[5,8]$, decreased significantly within 1 month, also remaining stable up to 3 months (data not shown). However, it remains to be investigated whether these outcomes remain stable in the long term.

Quality of life was also captured in this study during hospital visits at baseline, month 1 and month 3 . The St George's Respiratory Questionnaire improved significantly at 1 month, exceeding the reported minimal clinically important difference (table 1) [9, 10]. Interestingly, questionnaires that were more focused on general health status, such as the Short-Form Health Survey and King's Sarcoidosis Questionnaire general health status, showed either no or less pronounced changes during prednisone treatment in our study group than scores purely including physical symptoms such as the MRC, FAS and King's Sarcoidosis Questionnaire lung scores (table 1). This may have been caused by concurrent side-effects that occur during prednisone treatment, counterbalancing the positive outcomes of prednisone treatment. Indeed, in our study a number of adverse events were reported that are associated with prednisone treatment, including weight gain. Weight significantly increased within the first 3 months of treatment (mean increase $5.2 \pm 4.1 \mathrm{~kg}, \mathrm{p}<0.001)$. These data confirm that it remains relevant to continuously evaluate benefit-risk ratio of prednisone treatment in consultation with the individual patient $[2,11,12]$.

This study is the first to perform daily home spirometry in pulmonary sarcoidosis. Daily home spirometry could facilitate a personalised approach to treatment for each patient, aiming at achieving the maximum effect of lung function and symptom improvement with the lowest possible dose of prednisone, in order to minimise side-effects. This study shows that reliability of daily measured home spirometry in sarcoidosis patients is high; Pearson correlation between FVC measurements on the home spirometer and the in-hospital lung function equipment was $0.98(\mathrm{p}<0.001)$.

Together, our data argue that monitoring FVC changes at approximately 2-3 weeks after initiation of therapy, either at home or in clinic, can help physicians to better evaluate response to therapy in newly

\begin{tabular}{|c|c|c|c|c|}
\hline Symptom and/or QoL-related questionnaire & Patients & Baseline & Month 1 & Month $3^{\#}$ \\
\hline MRC score & 18 & $2.44 \pm 1.04$ & $1.33 \pm 0.91 *$ & $1.39 \pm 0.92 *$ \\
\hline FAS score & 18 & $27.4 \pm 10.8$ & $21.0 \pm 6.6^{*}$ & $20.7 \pm 7.6^{*}$ \\
\hline \multicolumn{5}{|l|}{ SGRQ } \\
\hline Symptoms & 17 & $45.0 \pm 21.1$ & $29.7 \pm 22.4^{*}$ & $27.5 \pm 25.1^{*}$ \\
\hline Activity & 17 & $55.9 \pm 24.9$ & $38.8 \pm 27.0 *$ & $36.8 \pm 28.4^{*}$ \\
\hline Impact & 17 & $28.4 \pm 23.5$ & $18.5 \pm 15.7$ & $18.2 \pm 15.1 *$ \\
\hline Total & 17 & $40.2 \pm 21.8$ & $26.5 \pm 18.1 *$ & $25.4 \pm 19.1 *$ \\
\hline \multicolumn{5}{|l|}{ SF-36 } \\
\hline PCS & 15 & $39.0 \pm 6.9$ & $40.2 \pm 6.9$ & $37.4 \pm 6.9$ \\
\hline MCS & 15 & $33.5 \pm 6.3$ & $36.2 \pm 7.0$ & $39.2 \pm 8.0^{*}$ \\
\hline \multicolumn{5}{|l|}{ KSQ } \\
\hline GHS & 11 & $61.9 \pm 14.5$ & $72.0 \pm 18.2 *$ & $67.0 \pm 15.0^{\text {ๆ }}$ \\
\hline Lung & 10 & $59.0 \pm 13.5$ & $70.4 \pm 23.1$ & $70.1 \pm 20.2^{*}$ \\
\hline
\end{tabular}

Data are presented as $\mathrm{n}$ or mean \pm SD. MRC: Medical Research Council; FAS: Fatigue Assessment Scale; SGRQ: St George's Respiratory Questionnaire; SF-36: Short-Form health survey; PCS: physical component score; MCS: mental component score; KSQ: King's Sarcoidosis Questionnaire; GHS: general health status.

${ }^{\#}$ : none of the values are statistically significant at month 3 compared with values at month $1 ;{ }^{\eta^{n}}: p=0.055$;

*: $p<0.05$ compared with baseline, using a paired sample t-test. 
treated sarcoidosis patients. Consequently, physicians might decide on earlier dose tapering and/or the need for initiation of second-line steroid-sparing therapies than is now advised [13, 14]. Future studies are needed to evaluate whether home monitoring of prednisone treatment (including FVC and symptom scores) and personalised dose titration [15] will allow for a non-inferior treatment effect compared to current clinical practice, while reducing side-effects and increasing the quality of life for patients with pulmonary sarcoidosis.

Caroline E. Broos ${ }^{1}$, Monique Wapenaar', Caspar W.N. Looman ${ }^{2}$, Johannes C.C.M. in 't Veen ${ }^{3}$,

Leon M. van den Toorn ${ }^{1}$, Maria J. Overbeek ${ }^{4}$, Marco J.J.H. Grootenboers ${ }^{5}$, Roxane Heller ${ }^{6}$, Rémy L. Mostard ${ }^{7}$, Linda H.C. Poell ${ }^{1}$, Henk C. Hoogsteden ${ }^{1}$, Mirjam Kool ${ }^{1}$, Marlies S. Wijsenbeek ${ }^{1,8}$ and Bernt van den Blink ${ }^{1,8}$

${ }^{1}$ Dept of Pulmonary Medicine, Erasmus MC, Rotterdam, The Netherlands. ${ }^{2}$ Dept of Public Health, Erasmus MC, Rotterdam, The Netherlands. ${ }^{3}$ Dept of Pulmonology, Franciscus Gasthuis \& Vlietland, Rotterdam, The Netherlands. ${ }^{4}$ Dept of Pulmonology, Haaglanden Medical Center, The Hague, The Netherlands. ${ }^{5}$ Dept of Pulmonary Diseases, Amphia hospital, Breda, The Netherlands. ${ }^{6}$ Dept of Pulmonology, Ikazia hospital, Rotterdam, The Netherlands. ${ }^{7}$ Dept of Respiratory Medicine, Zuyderland Medisch Centrum Heerlen, Heerlen, The Netherlands. ${ }^{8}$ These authors contributed equally.

Correspondence: Marlies S. Wijsenbeek, Dept of Pulmonary Medicine, Erasmus MC, 's-Gravendijkwal 230, 3015 CE, Rotterdam, Netherlands. Email: m.wijsenbeek-lourens@erasmusmc.nl

Received: Aug 052017 | Accepted after revision: Oct 262017

Support statement: C.E. Broos was supported by a Travel Award from the ATS PAR member Foundation for Sarcoidosis Research and by a Research enhancement grant from the Dutch sarcoidosis patient foundation (SBN). Funding information for this article has been deposited with the Crossref Funder Registry.

Conflict of interest: None declared.

This study was registered in the Dutch National Trial Register as NTR4328.

Acknowledgements: The authors gratefully acknowledge patients, research nurses, respiratory function technologists and physicians participating in this study from Erasmus MC, Franciscus Gasthuis \& Vlietland, Ikazia hospital, Amphia hospital, Haaglanden Medical Centre and Zuyderland Medical Center Heerlen (all the Netherlands). The authors thank Mirjam van Manen, Linda de Kleer, Frans Mertens and Hadassa de Raaf (Dept of Pulmonary Medicine, Erasmus MC, Rotterdam, the Netherlands) for technical assistance.

The authors contributed as follows. C.E. Broos, M. Wapenaar, J.C.C.M. in 't Veen, L.M. van den Toorn, M.J.J.H. Grootenboers, R. Heller, L.H.C. Poell, H.C. Hoogsteden, M.S. Wijsenbeek and B. van den Blink contributed to the study concept and design. C.E. Broos, M. Wapenaar, J.C.C.M. in 't Veen, L.M. van den Toorn, M.J.J.H. Grootenboers, R. Heller M.J. Overbeek, R.L. Mostard, L.H.C. Poell, H.C. Hoogsteden, M. Kool, M.S. Wijsenbeek and B. van den Blink, patient recruitment and data collection. C.E. Broos, M. Wapenaar, C.W.N. Looman, M.S. Wijsenbeek and B. van den Blink, data analysis and interpretation. C.E. Broos, M. Wapenaar, C.W.N. Looman, M.S. Wijsenbeek and B. van den Blink, manuscript preparation and manuscript drafting. All authors gave final approval on the manuscript.

\section{References}

Judson MA. The treatment of pulmonary sarcoidosis. Respir Med 2012; 106: 1351-1361.

Baughman RP, Lower EE. Treatment of sarcoidosis. Clin Rev Allergy Immunol 2015; 49: 79-92.

McKinzie BP, Bullington WM, Mazur JE, et al. Efficacy of short-course, low-dose corticosteroid therapy for acute pulmonary sarcoidosis exacerbations. Am J Med Sci 2010; 339: 1-4.

4 Goldstein DS, Williams MH. Rate of improvement of pulmonary function in sarcoidosis during treatment with corticosteroids. Thorax 1986; 41: 473-474.

5 Keir G, Wells AU. Assessing pulmonary disease and response to therapy: which test? Semin Respir Crit Care Med 2010; 31: 409-418.

6 de Kleijn WP, De Vries J, Wijnen PA, et al. Minimal (clinically) important differences for the Fatigue Assessment Scale in sarcoidosis. Respir Med 2011; 105: 1388-1395.

7 Baughman RP, Lower EE, Saketkoo LA. Clinical trials in pulmonary sarcoidosis. Curr Opin Pulm Med 2015; 21: 525-531.

8 Bargagli E, Mazzi A, Rottoli P. Markers of inflammation in sarcoidosis: blood, urine, BAL, sputum, and exhaled gas. Clin Chest Med 2008; 29: 445-458.

9 Swigris JJ, Brown KK, Behr J, et al. The SF-36 and SGRQ: validity and first look at minimum important differences in IPF. Respir Med 2010; 104: 296-304.

10 Jones PW. St. George's Respiratory Questionnaire: MCID. COPD 2005; 2: 75-79.

11 Wijsenbeek MS, Culver DA. Treatment of sarcoidosis. Clin Chest Med 2015; 36: 751-767.

12 Judson MA. Corticosteroids in sarcoidosis. Rheum Dis Clin North Am 2016; 42: 119-135, ix.

13 Statement on sarcoidosis. Joint Statement of the American Thoracic Society (ATS), the European Respiratory Society (ERS) and the World Association of Sarcoidosis and Other Granulomatous Disorders (WASOG) adopted by the ATS Board of Directors and by the ERS Executive Committee, February 1999. Am J Respir Crit Care Med 1999; 160: 736-755.

14 Bradley B, Branley HM, Egan JJ, et al. Interstitial lung disease guideline: the British Thoracic Society in collaboration with the Thoracic Society of Australia and New Zealand and the Irish Thoracic Society. Thorax 2008; 63: Suppl. 5, v1-v58.

15 Hashimoto S, Brinke AT, Roldaan AC, et al. Internet-based tapering of oral corticosteroids in severe asthma: a pragmatic randomised controlled trial. Thorax 2011; 66: 514-520. 